Supporting Information

\title{
Sequential inactivation of gliotoxin by the $S$-methyltransferase TmtA
}

Elke R. Duell, ${ }^{\ddagger}$ Manuel Glaser ${ }^{\ddagger}$ Camille Le Chapelain, Iris Antes, Michael Groll and Eva M. Huber*

Center for Integrated Protein Science Munich (CIPSM) at the Department Chemie, Technische Universität München, Lichtenbergstr. 4, 85748 Garching, Germany

${ }^{\ddagger}$ E.R.D. and M.G. contributed equally to this work.

*Email: eva.huber@tum.de

Table of contents

1. Supporting Experimental Procedures $\quad$ S2

2. Supporting Figures S6

$\begin{array}{ll}\text { 3. Supporting Tables } & \text { S17 }\end{array}$

4. Supporting References $\quad$ S20 


\section{Supporting Experimental Procedures}

\section{Computational Studies}

Molecular Docking

Molecular protein-ligand docking calculations were performed using the Flex $\mathrm{X}^{1}$ and FlexXPharm $^{2}$ methods as implemented in the LeadIT drug design platform ${ }^{3}$ for docking differently methylated derivatives of gliotoxin into the binding site of TmtA. The 2D ligand structures were initially generated with ChemBioDraw Ultra $13.0^{4}$ and subsequently converted to a $3 \mathrm{D}$ structural ligand model in Tripos Mol2 file format with Chem3D Pro 13.0. ${ }^{5}$ These structural models were used as ligand input files for FlexX. The TmtA:SAM ( $S$-(5'-adenosyl)-Lmethionine (SAM)) complex was modeled by adding a methyl group to the bound SAH ( $S$ (5'-adenosyl)-L-homocysteine) cofactor in the biologically active $S$ configuration at the sulfur atom using PyMOL. ${ }^{6}$ Hydrogen atoms of the coenzymes SAH and SAM were placed with Open Babel 2.2.3. ${ }^{7}$ The mutations M10A, F11A, F16A and Y20A to create the quadruple TmtA mutant were inserted into the TmtA crystal structure by applying the IRECS side chain prediction method together with the ROTA potential. ${ }^{8,9}$ Amino and carboxy-groups of the homocysteine and methionine moieties of SAH and SAM were considered in their protonated and unprotonated form, respectively. Spatial constraints were defined during docking of reduced gliotoxin, mono(methylthio)gliotoxin isoforms and bis(methylthio)gliotoxin to SAHand SAM-bound TmtA structures to ensure ligand conformations able to participate in the enzymatic reaction. To this end, the thiol- or methylthio-group of the ligand was required to be located in a $4 \AA$ radius sphere centered on a key atom of the coenzyme (i.e. the $\mathrm{C}$ atom of the SAM coenzyme methyl group or the $\mathrm{S}$ atom of the SAH coenzyme). All FlexX dockings were performed using standard conditions except for docking of 3-SH mono(methylthio)gliotoxin into the SAM:TmtA complex and for docking of bis(methylthio)gliotoxin into the SAH:TmtA complex, for which the maximum allowed overlap volume for protein-ligand clashes was raised from $2.9 \AA^{3}$ to $4 \AA^{3}$ and $3 \AA^{3}$, respectively. In all docking calculations, amino acids within a radius of $20 \AA$ of SAM were included into the active region of the molecular docking calculations and default amino acid assignments were accepted. The binding affinity of the coenzyme:TmtA complexes was estimated with the FlexX scoring function using the ANALYZE functionality of the FlexX shell in the LeadIT command-line mode. The coenzyme molecules were protonated as under 
physiological conditions for the computation of the FlexX score and the active site radius was set to $20 \AA$.

\section{$\underline{\text { Molecular dynamics }}$}

The docked ligand:coenzyme:TmtA complexes were subsequently energy minimized using the AmberTools14 and Amber14 software packages ${ }^{10}$ in order to remove any overlap between protein and ligand, and to relax the docked complex models. Additionally, the SAH:TmtA and SAM:TmtA complexes were minimized. The Amber03 force field parameter set ${ }^{11}$ was used for the TmtA protein and the GAFF force field parameter set ${ }^{12}$ was used for SAM, SAH, and the different gliotoxin derivatives. The partial charges for the atoms of SAM, SAH, and the gliotoxin derivatives were derived with the PyRED server ${ }^{13}$ using standard settings ("RESP-A1" charge model, HF/6-31G(d) level). GAFF atom types were assigned with the antechamber utility. ${ }^{14}$ Missing parameters were estimated with the parmchk tool. ${ }^{14}$ The docked complexes and the two coenzyme:TmtA complexes were prepared for minimization with the tleap utility, ${ }^{15}$ which modelled the hydrogen atoms and termini of the TmtA protein crystal structure. All energy minimizations were conducted in a neutralized, rectangular TIP3P ${ }^{16}$ water box extending at least $12 \AA$ from any protein atom at each side of the box. Energy minimizations were performed with sander or pmemd.MPI. For every complex, two subsequent minimizations were conducted. First, 10,100 steps of restraint minimization (100 steps with the steepest descent algorithm and 10,000 steps with the conjugate gradient method) were done with the TmtA atoms restrained using a $50 \mathrm{kcal} \cdot \mathrm{mol}^{-1} \cdot \AA^{-2}$ force constant. Second, 100,100 steps of energy minimization (100 steps with the steepest descent algorithm and 100,000 steps with the conjugate gradient method) were conducted without restraints. Both minimizations were considered as converged, if the root-mean-square of the Cartesian components of the energy gradient was less than $0.0001 \mathrm{kcal} \cdot \mathrm{mol}^{-1} \cdot \AA^{-1}$. The non-bonded interaction cut-off was set to $8.0 \AA$ for both energy optimizations. Afterwards the system was equilibrated according to the protocol given in Table S4. At each equilibration step the systems initial velocities were randomly assigned from a Maxwell-Boltzmann distribution at the given target temperature. After equilibration of the systems, $5 \mathrm{~ns}$ of MD were performed as basis for the MM-PBSA and MM-GBSA calculations. MD simulations were performed with 1 fs time steps. Non-bonded interactions were computed applying a cut-off of $14 \AA$. The Particle Mesh Ewald method was used to calculate long-range electrostatic interactions. ${ }^{17}$ The SHAKE algorithm ${ }^{18}$ was used to constrain bonds to hydrogen atoms. The Berendsen 
thermostat $^{19}$ was applied with a time constant of 1 ps to ensure constant temperature. The Berendsen barostat ${ }^{19}$ was applied with a compressibility of $45 \times 10^{-6}$ bar $^{-1}$ and a pressure relaxation time of 1 ps to keep a constant target pressure of 1 bar. All MD simulations were performed using the pmemd.CUDA program ${ }^{20}$ from the Amber14 software package ${ }^{10}$.

\section{$\underline{\text { MM-PBSA and MM-GBSA calculations }}$}

To estimate the free energy of binding of the different gliotoxin compounds to the corresponding TmtA:coenzyme complexes and the free energy of binding of the cofactors SAH and SAM to the TmtA protein, Molecular Mechanics - Poisson-Boltzmann Surface Area (MM-PBSA) $)^{21-23}$ and Molecular Mechanics - Generalized Born Surface Area (MMGBSA $)^{22}$ calculations were performed, according to equations 1 and 2 , based on a single MD simulation of the complexes. Frames for snapshot ensembles were collected every 10 ps from the last 2 ns of a $5 \mathrm{~ns}$ long production run. MM-PBSA and MM-GBSA calculations were conducted applying the MMPBSA.py script ${ }^{24}$ from the AmberTools14 software package. ${ }^{10}$ $\mathrm{G}_{\text {complex }}, \mathrm{G}_{\text {receptor }}$ and $\mathrm{G}_{\text {ligand }}$ (cf. equation 1) represent the approximated free energies of the complex, receptor, and ligand, respectively, and the total free energy of binding was calculated according to eq. 1 :

$$
\Delta G_{\text {bind }}=G_{\text {complex }}-G_{\text {receptor }}-G_{\text {ligand }}
$$

The free energies of the individual components were approximated as sum of the ensembleaverages of (i) the molecular mechanics potential energy $\mathrm{E}_{\mathrm{MM}}$, (ii) an estimate of the polar part of the solvation free energy $\mathrm{G}_{\text {polar-solvation }}$ and (iii) an estimate of the apolar part of the solvation free energy $\mathrm{G}_{\text {apolar-solvation: }}$

$$
G_{\text {component }}=\left\langle E_{M M}\right\rangle+\left\langle G_{\text {polar-solvation }}\right\rangle+\left\langle G_{\text {apolar-solvation }}\right\rangle
$$

Explicit approximations of entropic contributions to the free energy of binding were neglected. The estimate of the polar term of the solvation free energy was calculated applying the linear Poisson-Boltzmann equation together with the optimized radii by Tan and Luo ${ }^{25}$ and default settings or via the Generalized Born (GB) OBC-model developed by Onufriev et $a l .{ }^{26}$ together with the radii set 'mbondi2' (cf. Amber14 ${ }^{10}$ ) and default settings. The estimate of the apolar part of the solvation free energy was approximated via the solvent-accessible surface area (SASA) applying a surface tension of $0.0072 \mathrm{kcal} \cdot \mathrm{mol}^{-1} \cdot \AA^{2}{ }^{27}$ The SASA term was computed with the LCPO (Linear Combination of Pairwise Overlaps) ${ }^{28}$ method using a 
probe radius of $1.4 \AA$ and the 'mbondi2' radii set (cf. Amber $14{ }^{10}$ ). For the calculations of the free energy of the receptor, the coenzyme was considered as part of the receptor, except for the approximations of the free energy of SAM and SAH binding, where SAM and SAH were considered as the ligand.

Note: Using the MM-PBSA and MM-GBSA methods, energies within the physically reasonable range are obtained (Table 1). In this context, it has to be stated that the MM-PBSA and MM-GBSA methods tend to overestimate absolute binding free energies by a factor of 10 , but provide very accurate results for relative binding free energies of different ligands to the same protein (up to $\pm 1 \mathrm{kcal} \mathrm{mol}^{-1}$ ). Thus the overall binding free energy values for the gliotoxin ligands should be in the range of -3 to $-5 \mathrm{kcal} \mathrm{mol}^{-1}$. 


\section{Supporting Figures}

a

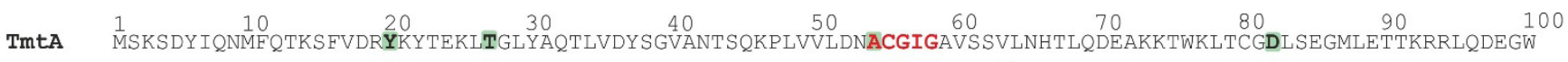

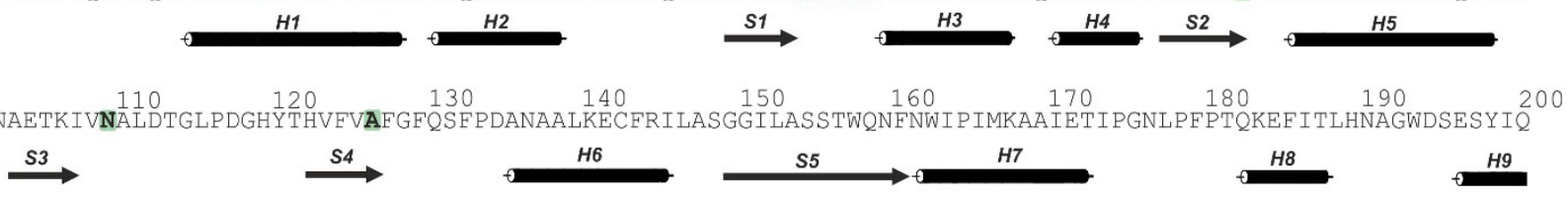

TmtA VNAETKIVNALDTGLPDGHYTHVEVAFGFQSFPDANAALKECFRILASGGILASSTWQNFNWIPIMKAAIETIPGNLPFPTQKEFITLHNAGWDSESYIQ

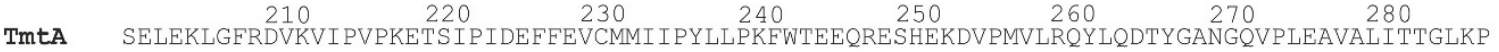

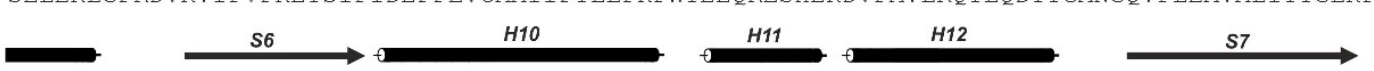

b
A. fumigatus
A. clavatus
A. flavus
A. niger
A. nomius
A. terreus
N. fischeri
N. udagawae
P. chrysogenum
P. oxalicum

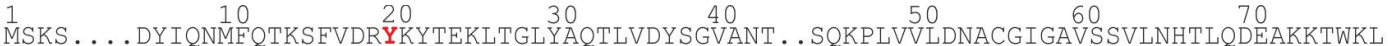
MTKI .... DYLQEMFQRKDFVDRYKYSERLTGMYAQTLVDFSSVAQP . . GQKPLVVLDNACGLGAVSSALQHTLDDEAKRNCHL MQESLTSGSSAS LNIHDKRFAAL YELGGKITELFAKELISQSGLPWS . . SQEPLVVLDNACGTGAVSSVLHHT I GNDKKANWHL MTPKI . . . . . SPSSYLTPAFVSRYHLAEKLTGVFVAPLVQHSGILS. TPSNKPMAVFDNACGLGIVSSYLNSTLPEDVKRHWTL MHEALTIDRPASQNVHDSRFAALYELDGGITELFAKELISQSGLALS . . SHDPVVI LDNACGTGAVSSVLHRTIGSDKKGNWQL

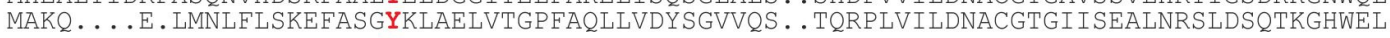
MSKI . . . . NY IQNMFQTKDFVDRYKYTEKLTGLYAQTLVDYSGVANT . . SQKPLVVLDNACGIGAVSSVLNRTLDDEAKKTWKL ML . . . . . . EKVFREKSFADQYTYGAKISELYAETLVDRSGIAKS . .HQRPLI IFDNACGTGS ISSTLQRTLDKRNKRSLKL MTRA... . TNFTELYAGKGILDTYMVAEKITRYYTQDLIQLSGLSES . . SLTPLVILDLACGTGVVSDALHDMLNFQPKGNWEL MAANT . . KGWEKIFKDDQFVKQYKTGEH ITGRFARILLDQSGLLTNSS PDTSLIVFDNACGTGVVSS I LQSELDDQVKRKMQL

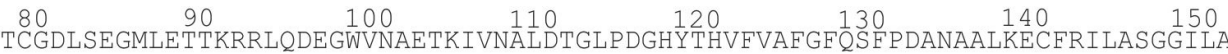
. TCGDITELMVEYTKLRIEREGWVNAEAKVVDAQCTGLPADKYTHVLTAFAFMMI PDARAAMRECFRILQSGGVLAT STWR. . . . TCGDMSDSMLYYTRQKMLQEEWHNTEVKIVNAQNTCLPSAHYTHVFTAFAFNLFPDDFSAMKGSRNASEYFNLV . . AFWQEPRG TCGDISDSLVOYVNORIODEGWPRAKAOLVDAODTKLPSSHFTHIFAAF . . . . . . . . ECLRILOPGGTVAISNWO . . . TCGDLSEGMLEYTKQRLQDEGWVNAETKIVNALDTGLPDAHYTHVFVSFGFQSFPDANAALKECFRILASGGILATSTWQ . . . . TCGDLSEGMVEYTKQRMQAEGWDNAEAKIVNAQDTGLPSDHYTHVYTAFAFNMFP DYKAALQECFRVLQPGGTLATSTWQ . . . . TCGDISTELTGHVKQK I LERGWENS IAKVVDAQNTELPTGHYTHVFAALAFTSFPDTYAAMKEVMRILQPGGTLTISTWQ . . . . TCGDISPGMLEYAKARADEEGWQNAHEEI IDAQQSGLPSSHFTHVIASFVYMALPKSLAALDDATRILRPGGTLAFSTWI . . . .

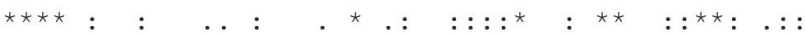
A. fumigatus
A. clavatus
A. flavus
A. niger
A. nomius
A. terreus
N. fischeri
N. udagawae
P. chrysogenum
P. oxalicum
A. fumigatus
A. clavatus
A. flavus
A. niger
A. nomius
A. terreus
N. fischeri
N. udagawae
P. chrysogenum
P. oxalicum

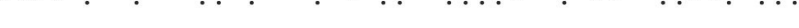

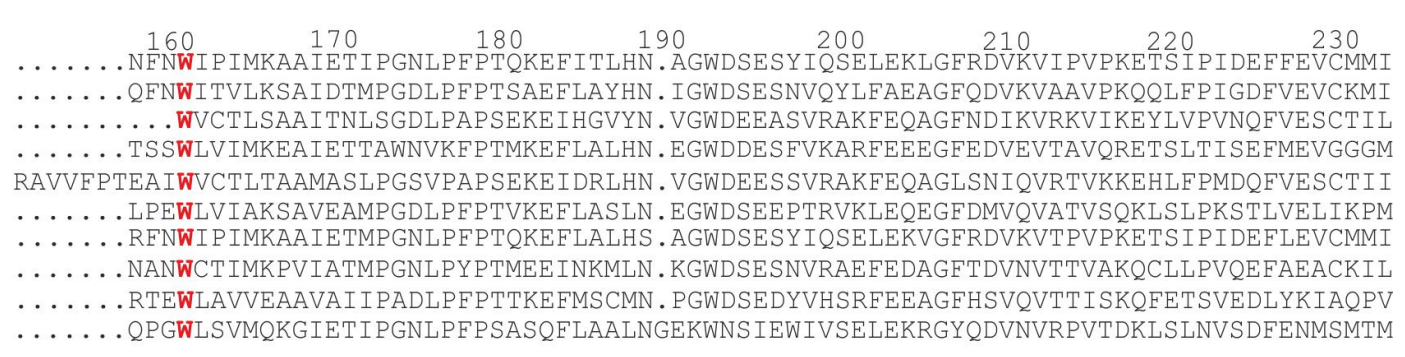

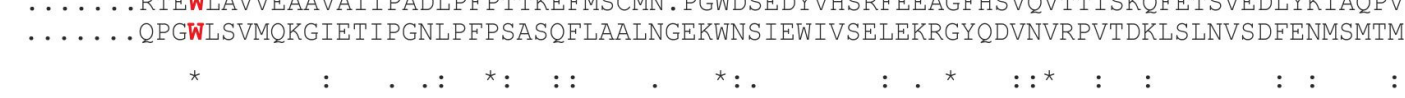

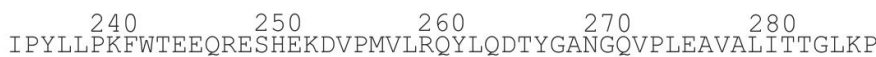
LPFVLSKVWTKEQRELYEKDVPAVLLRYLEETYGKDGQVSLEAVALITTGCKP IPTIVNI FWTQDQRDQYESELPMAVHRYVEGKYGRDGMASMEAEAIIATGHKH IPIVTGAFWTPEQREKYEAKAPVVIREYLEEKFGADGVIRMEPVAVLAVGRKP IPMIVNTFWTQDQRDQYESQLPLAIRRYAEGKFGKDGLASLEAEAIIATSRKP IPYLLPKFWTEEQRESHEKDVPMVLRQYLQDNYGANGQVPLEAVALITTALKS LPYVLSKFWTQEQRGRYEAEVPEHLMGYLEKAYGKDGLAPMKGVAI IATGRKP IPI IVSKWWNQEQRDKYENDI LPALQRHLNETYGENGLVPQEWTAVFATGQKGS FPMVTKAFWTEEQRKYSLDEVRLALRNYLQNTYGHEGDIPMQWTAIMATARKPN

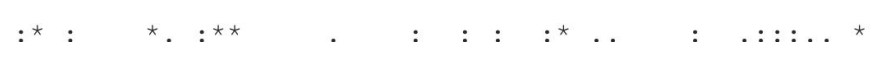
LPVILGRFWTDEQRAKHEKH I PTALQQYLDDKYGASDDVPVEPRVIIATARKPC 


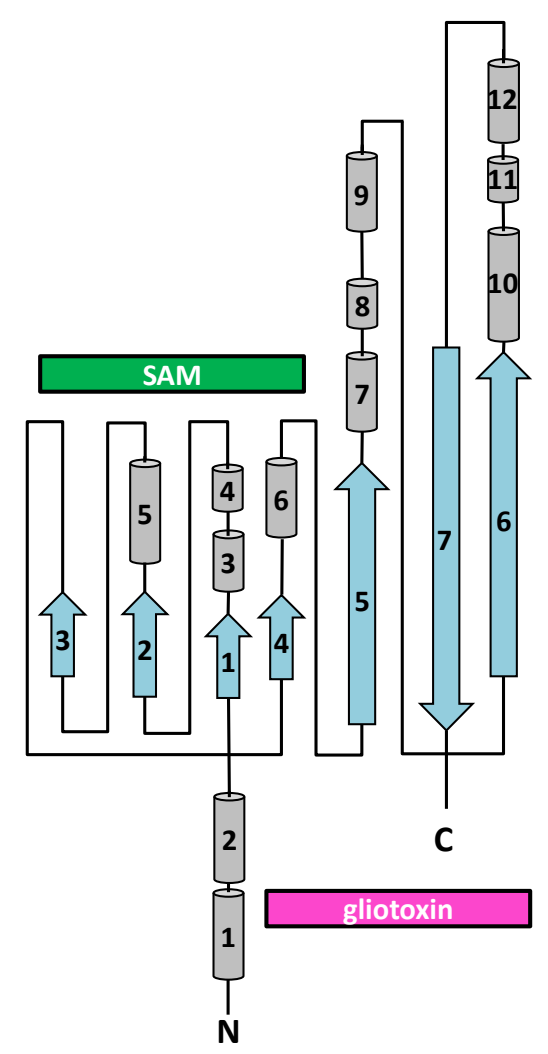

Supporting Figure S1. Sequence and structure of TmtA. a) Primary sequence of TmtA. Amino acids are numbered and secondary structures are assigned. The AxGxG nucleotide binding motif is coloured in red, amino acids engaged in cofactor binding are presented in bold and highlighted in green. b) Sequence alignment of Aspergillus fumigatus TmtA and its closest homologues according to blast search. The amino acids Tyr20 and Trp162, highlighted in red, are conserved. Abbreviations: Aspergillus (A.), Neosartorya (N.) and Penicillum (P.). c) The topology plot for TmtA indicates that the $\beta$-strands (blue) adopt the 3214576 order typical of SAM-dependent methyltransferases. The N-terminal part of the protein binds the cofactor, while the substrate binding pocket is formed mainly by the C-terminal part of the protein and the very $\mathrm{N}$-terminus. 

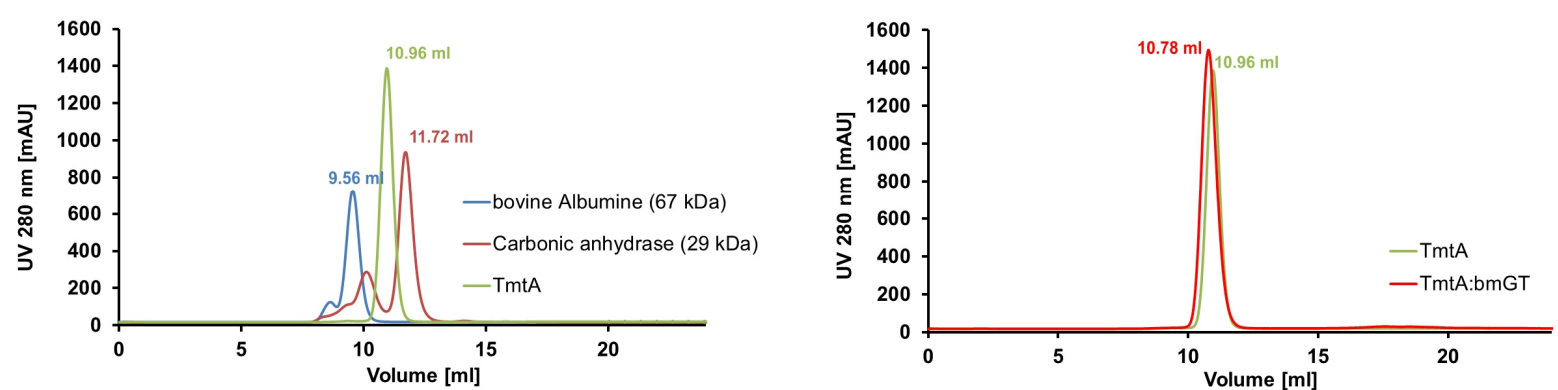

C
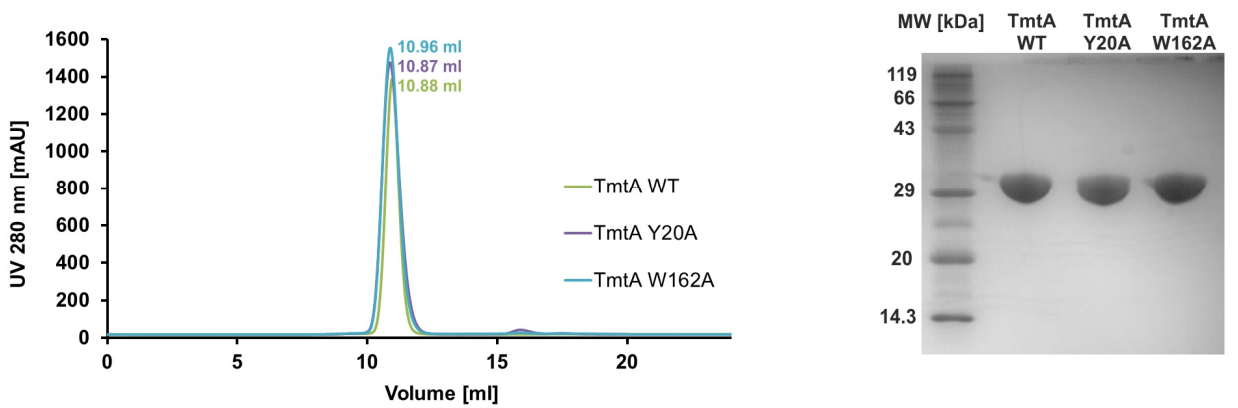

e

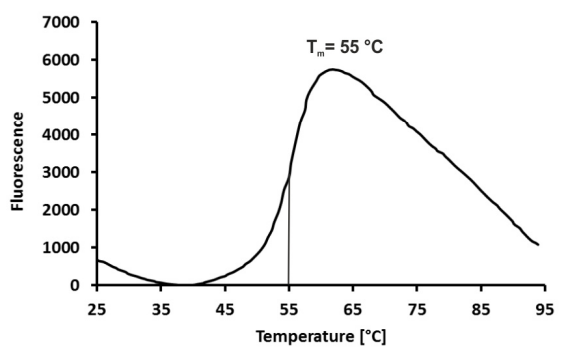

Supporting Figure S2. Purification of WT and mutant TmtA. a) Typical size exclusion chromatogram of WT TmtA under non-reducing conditions using buffer C (column: Superdex $7510 / 300 \mathrm{GL}, 24 \mathrm{ml}$ ). The retention volume of approximately $10.96 \mathrm{ml}$ corresponds to a molecular weight of about $39 \mathrm{kDa}$, as determined by calibration with standard proteins. The deviation from the calculated size for monomeric TmtA of $32.6 \mathrm{kDa}$ may result from its rodlike shape and the associated larger hydrodynamic radius compared with globular proteins of the same size. The elution profiles for carbonic anhydrase (size of $29 \mathrm{kDa}$ corresponds to a TmtA monomer) as well as bovine albumin (size of $67 \mathrm{kDa}$ corresponds to a TmtA dimer) are shown for comparison. b) Size exclusion chromatograms of WT TmtA in the absence or presence of bis-thiomethylated gliotoxin (bmGT). The compound does not affect the retention volume and thus the oligomerization state of TmtA. c) Size exclusion chromatograms of 
mutant TmtA are indistinguishable from the WT. d) SDS-PAGE analysis of WT and mutant TmtA after size exclusion chromatography confirms the purity of the proteins. e) Typical thermal shift assay diagram of TmtA in the presence of $5 \mathrm{mM}$ SAM. The melting temperature ranges between 50 and $55^{\circ} \mathrm{C}$ depending on the buffer used and is indicative of a stably folded protein.

a

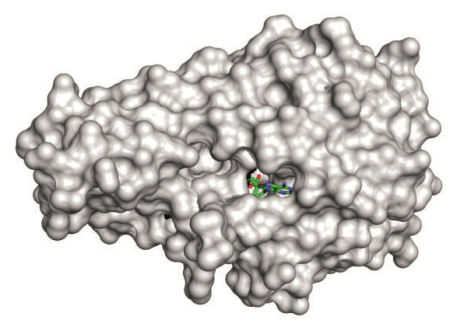

b

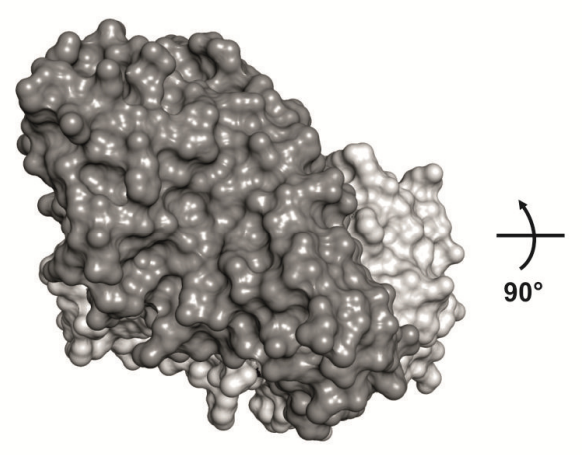

C

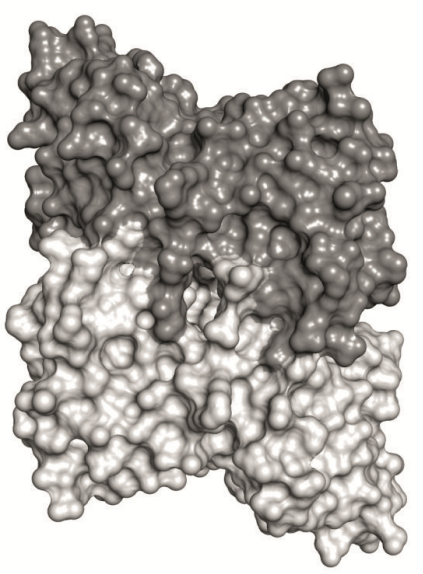

Supporting Figure S3. TmtA forms a homodimer in the crystal structure. a) Surface representation of a TmtA monomer (light gray) with the SAH cofactor (green) shown as balland-sticks. b) The second TmtA copy (dark gray) that is present in the asymmetric unit of the crystal lattice sits on top of the monomer shown in panel a (same orientation) and buries the two active sites. c) $90^{\circ}$ rotation of panel b. 
a
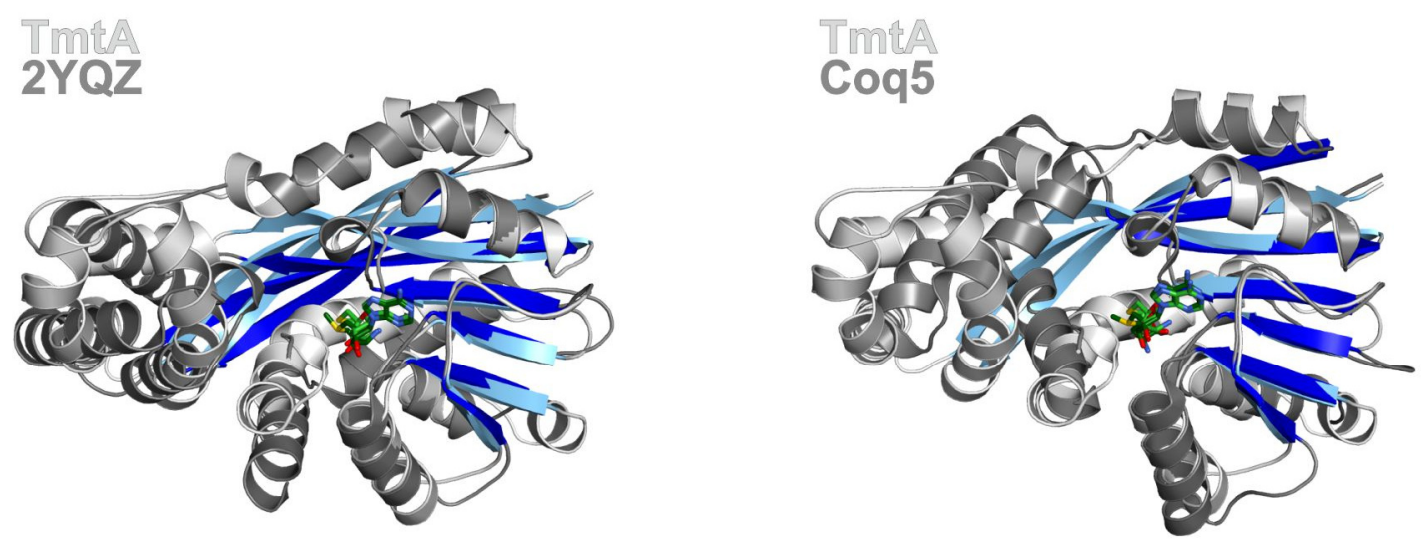

b

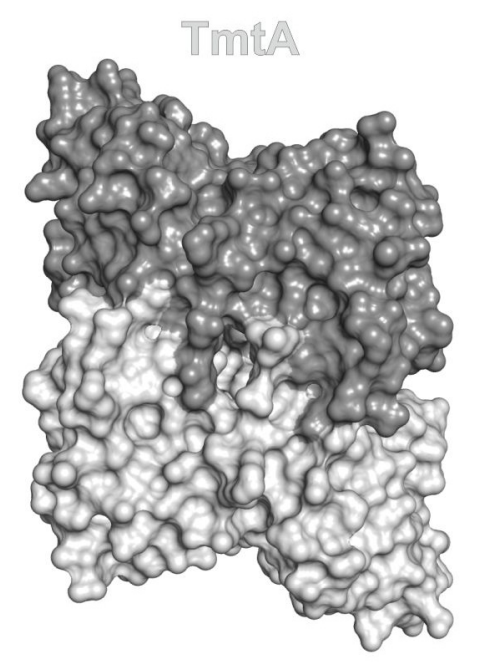

\section{Cog 5}

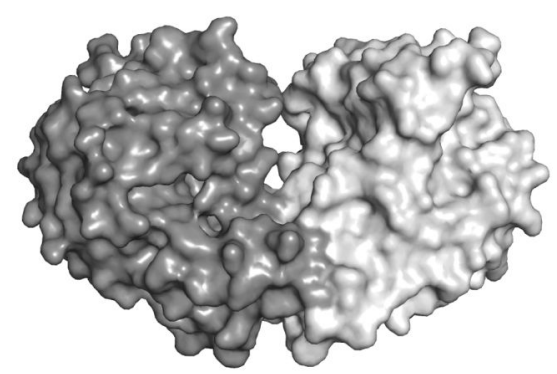

C

TmRA

2YQZ

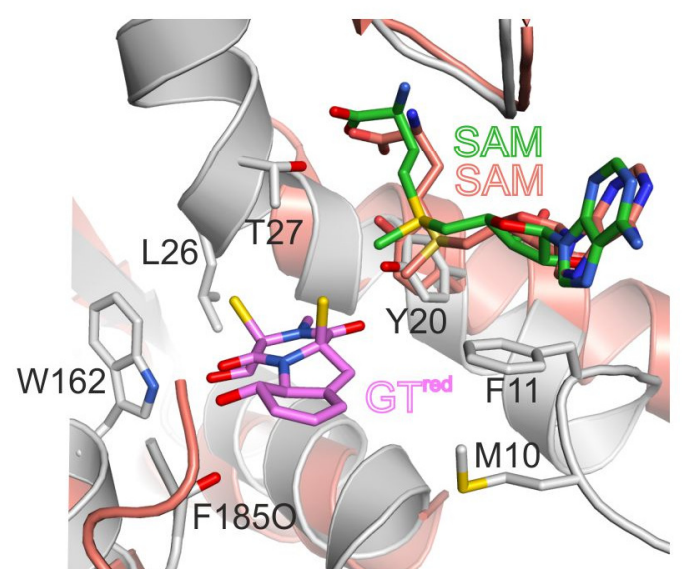

Tnits

Coq5

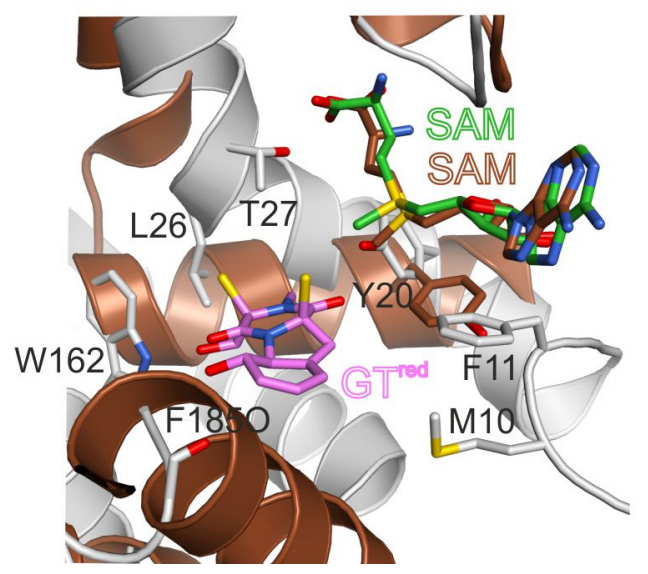

Supporting Figure S4. TmtA and structurally related MTs. a) Structural superpositions reveal that the hypothetical MT TTHA0223 from Thermus thermophilus (PDB entry code: 
2YQZ (RIKEN Structural Genomics/Proteomics Initiative); left, dark gray/blue) and the Coq5 monomer from Saccharomyces cerevisiae (PDB entry code: $4 \mathrm{OBW} ;^{29}$ right, dark gray/blue) adopt a fold similar to TmtA (light gray/blue). For details (e.g. r.m.s.d. values) see Table S2. Coq5 catalyzes the $C$-methylation in the biosynthesis of coenzyme $\mathrm{Q}$. b) The dimeric assemblies of TmtA and Coq5 significantly differ from each other. c) Structural superpositions of TmtA (gray) with 2YQZ (salmon; left) and Coq5 (PDB entry code: $4 \mathrm{OBW}{ }^{29}$ right; brown). Although the SAM moieties superimpose well, the active site of TmtA significantly differs from that of 2YQZ and Coq5. Due to different orientations and positions of secondary structure elements most amino acids engaged in binding of reduced gliotoxin $\left(\mathrm{GT}^{\mathrm{red}}\right.$, magenta) cannot be provided by 2YQZ or Coq5. Solely Tyr20 adopts a similar position in all structures.

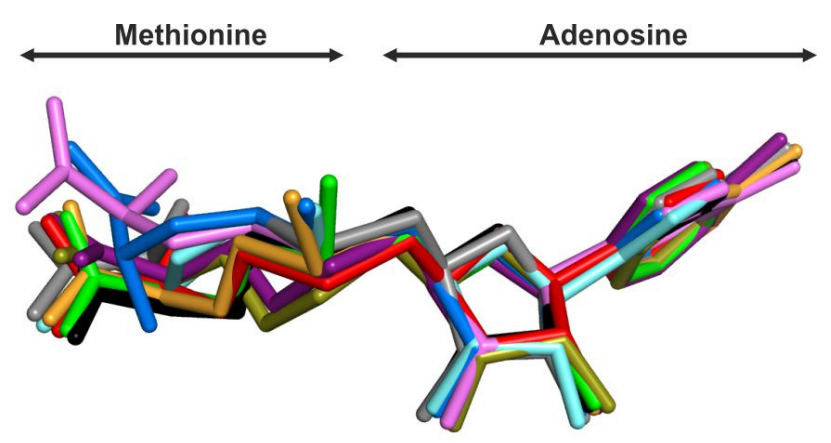

Supporting Figure S5. Superposition of SAH, SAI (S-(5'-adenosyl)-Lhomoselenocysteine) and SAM molecules bound to methyltransferases homologous to TmtA. The SAH cofactor bound to TmtA is shown in black; 2YQZ:SAM (RIKEN Structural Genomics/Proteomics Initiative; green), 4OBW:SAM ${ }^{29}$ (blue), 4KRI:SAM ${ }^{30}$ (red), 1M6E:SAH ${ }^{31}$ (rose), 1IM8:SAI ${ }^{32}$ (gray), 3DLC:SAM (Joint Center for Structural Genomics; yellow), 2GLU:SAM (New York SGX Research Center for Structural Genomics; cyan), 2P35:SAH (Midwest Center for Structural Genomics; purple), 3G5T:SAH (Center for Eukaryotic Structural Genomics; olive). The adenosine moieties perfectly align to each other, while the methionine residue can adopt slightly different conformations depending on the protein environment. For PDB entries see Table S2. 
a

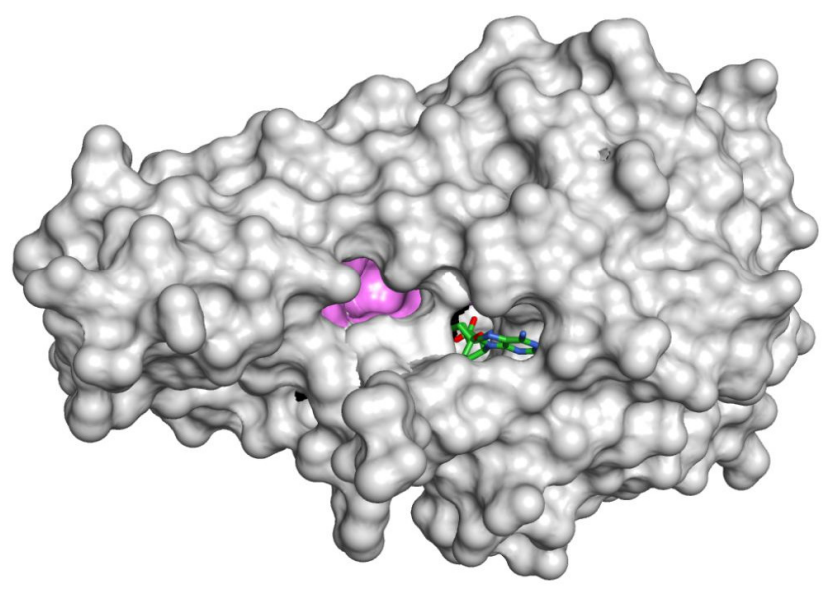

b

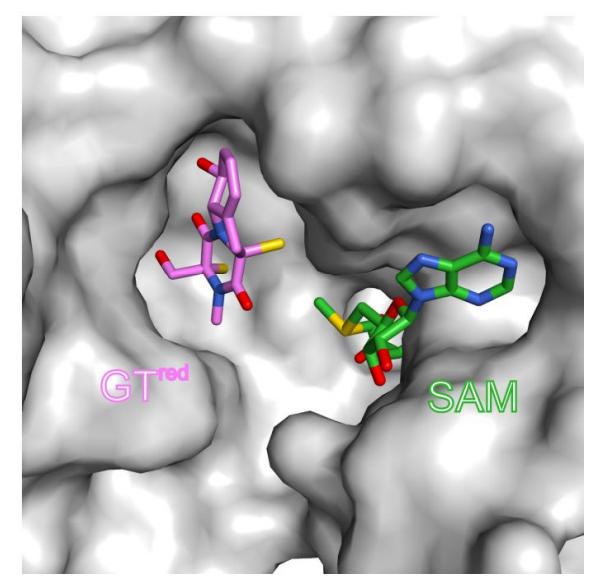

Supporting Figure S6. Surface illustration of TmtA. a) TmtA in the apo state; the cofactor $\mathrm{SAH}$ is depicted as green sticks. The well-shaped substrate binding pocket is coloured in magenta. b) A close-up view of the active site of TmtA bound to reduced gliotoxin $\left(\mathrm{GT}^{\mathrm{red}}\right)$ and SAM (docking results) illustrates the exquisite shape complementarity of the natural product and the substrate binding pocket. For clarity, the N-terminus (up to residue 20) and the amino acids 83,237 and 241 are not displayed. 

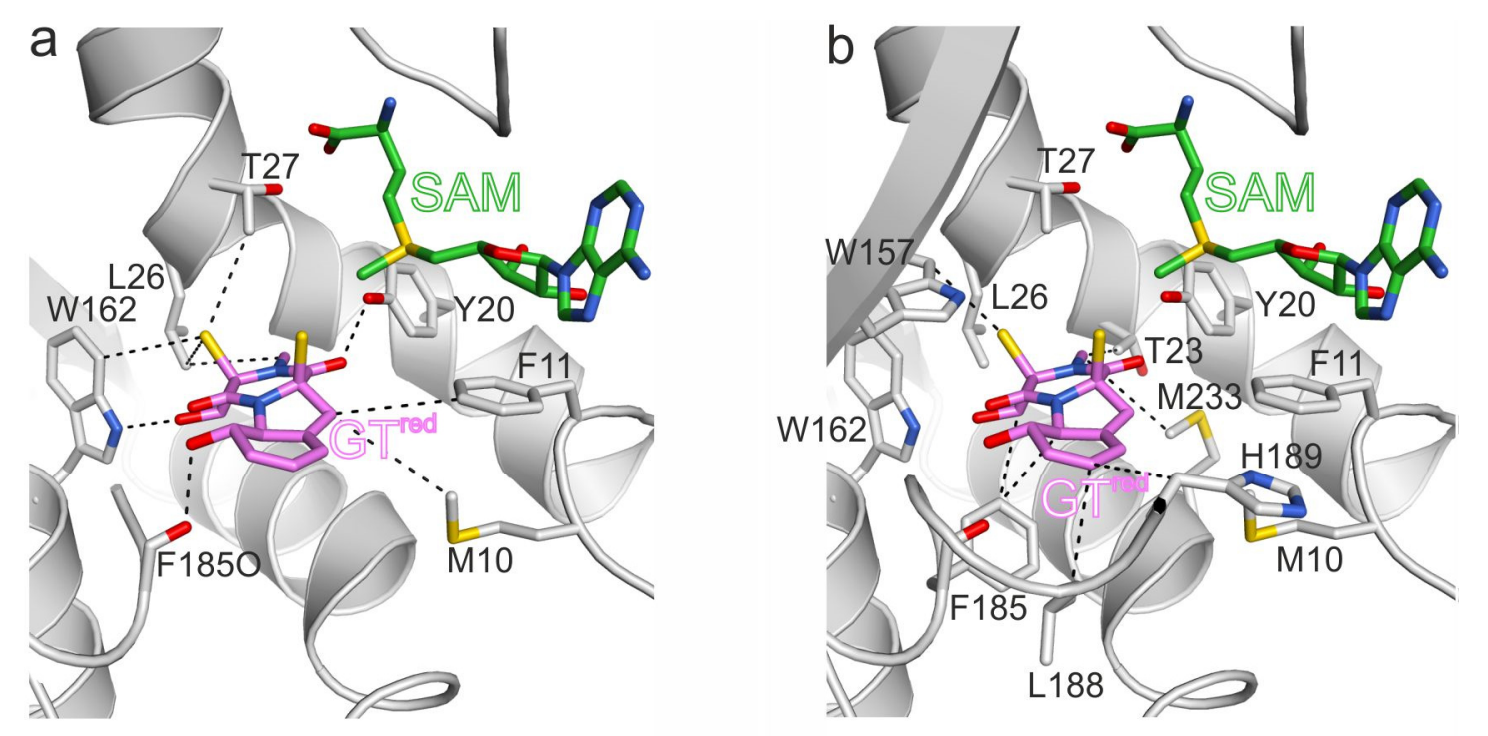

C

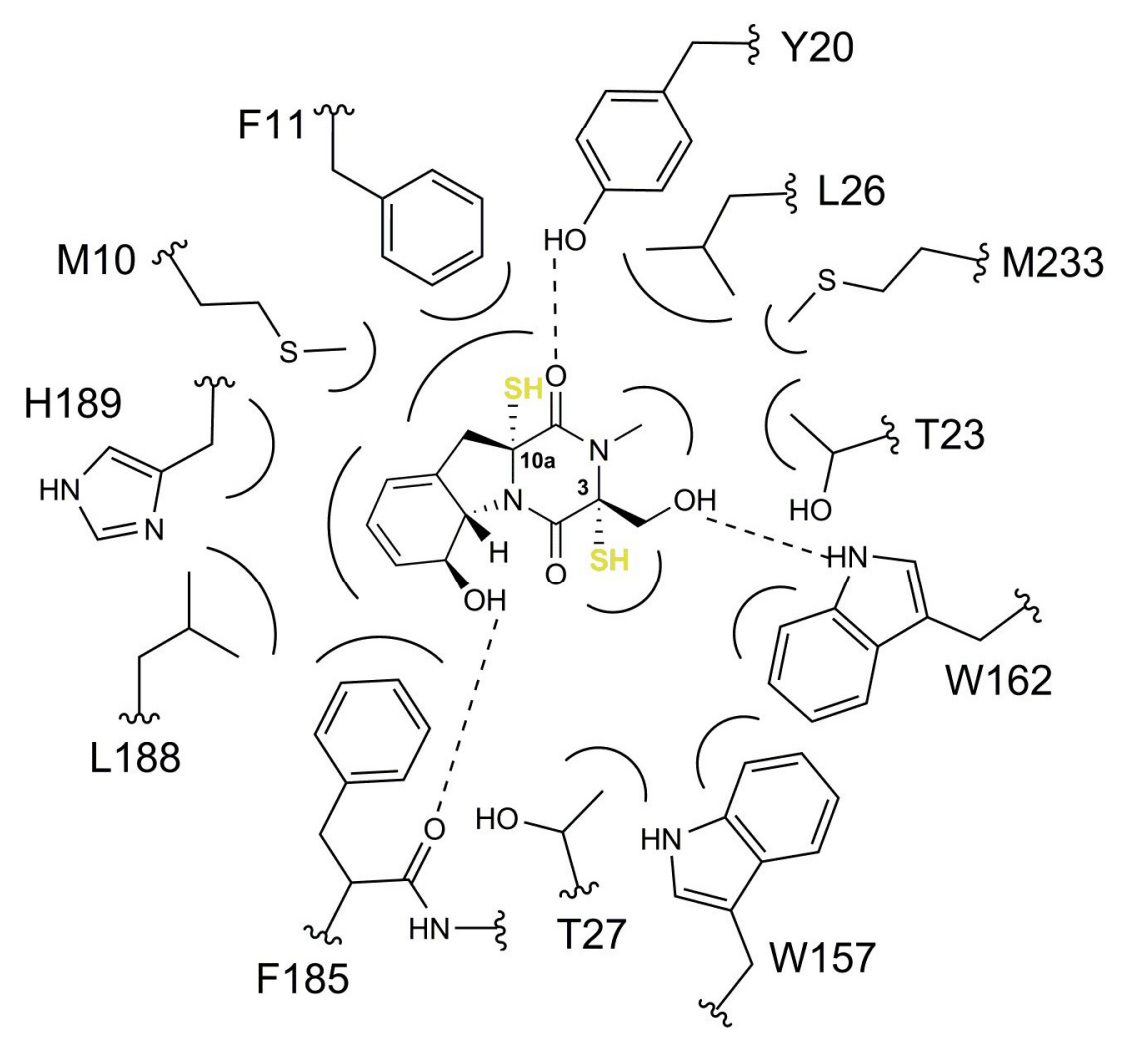

Supporting Figure S7. The active site of TmtA. a) Important amino acids involved in substrate binding are illustrated according to Figure 3a. b) Additional hydrophobic proteinsubstrate interactions are depicted by dashed black lines. For clarity, interactions shown in panel a are not displayed. c) Schematic plot of the interactions of reduced gliotoxin with the protein surroundings. 


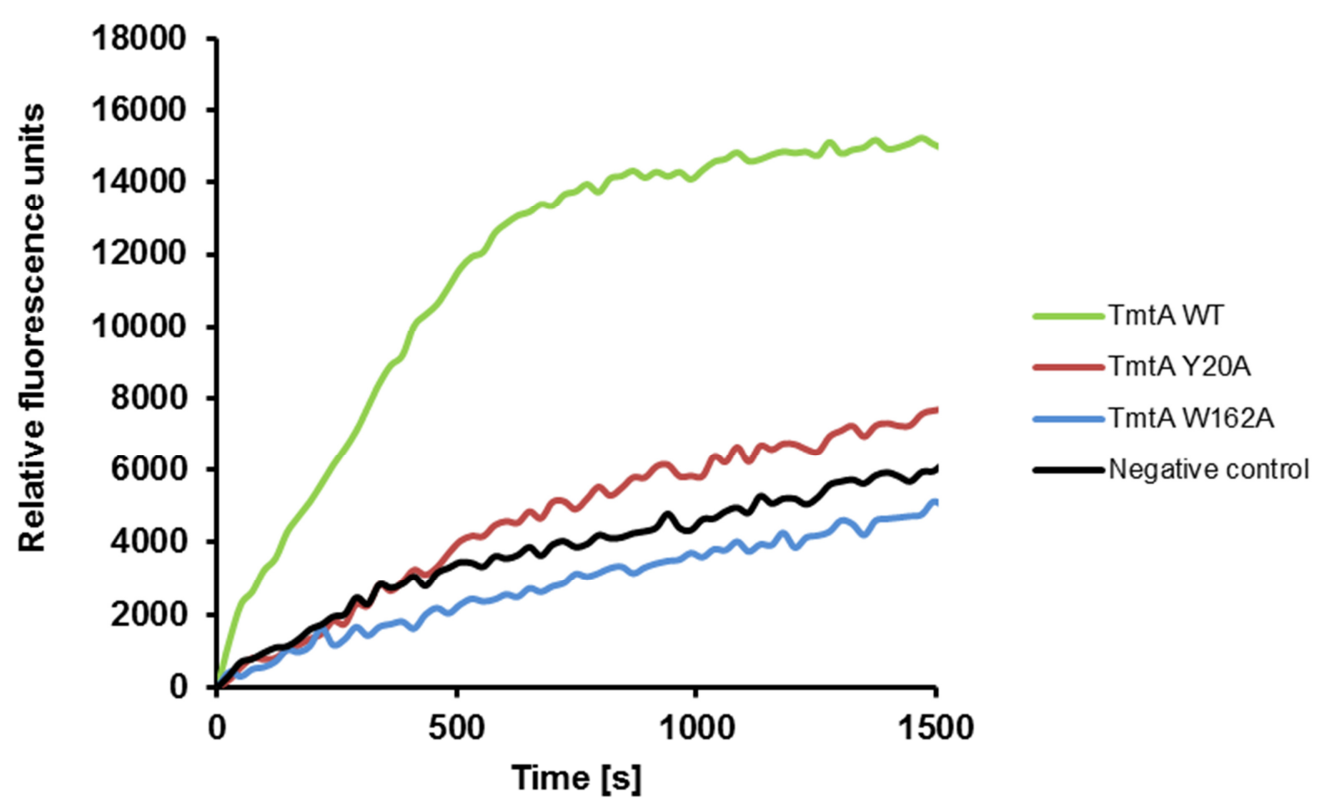

Supporting Figure S8. Enzymatic activity of TmtA. Relative enzymatic activity of WT and mutant TmtA as determined with the SAMfluoro Methyltransferase Assay (G-Bioscience). The final concentrations of enzyme, reduced gliotoxin and SAM were $1 \mu \mathrm{M}, 600 \mu \mathrm{M}$ and $166 \mu \mathrm{M}$, respectively. Note that enzymatic activity could only be detected with freshly reduced gliotoxin. 

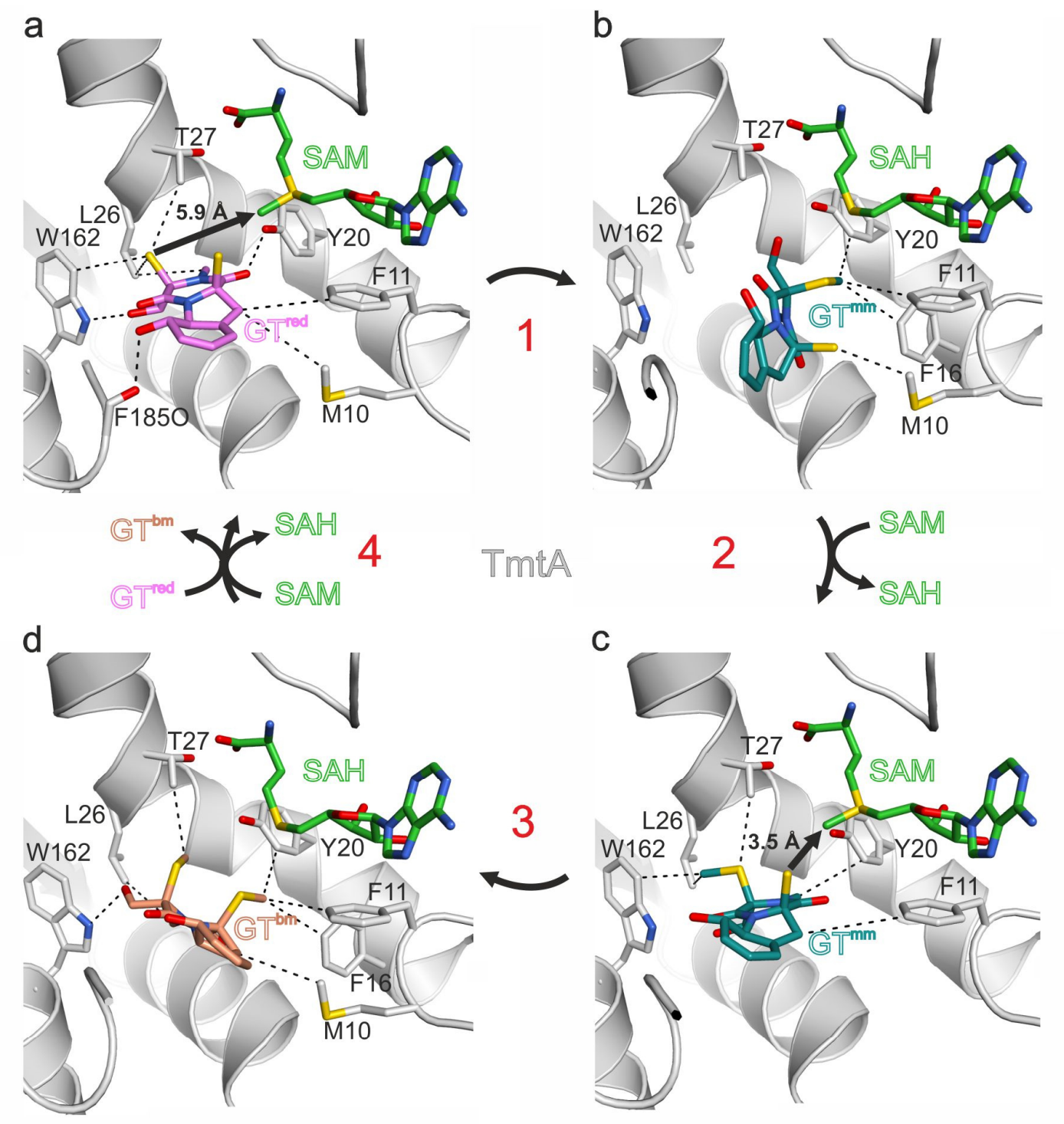

e
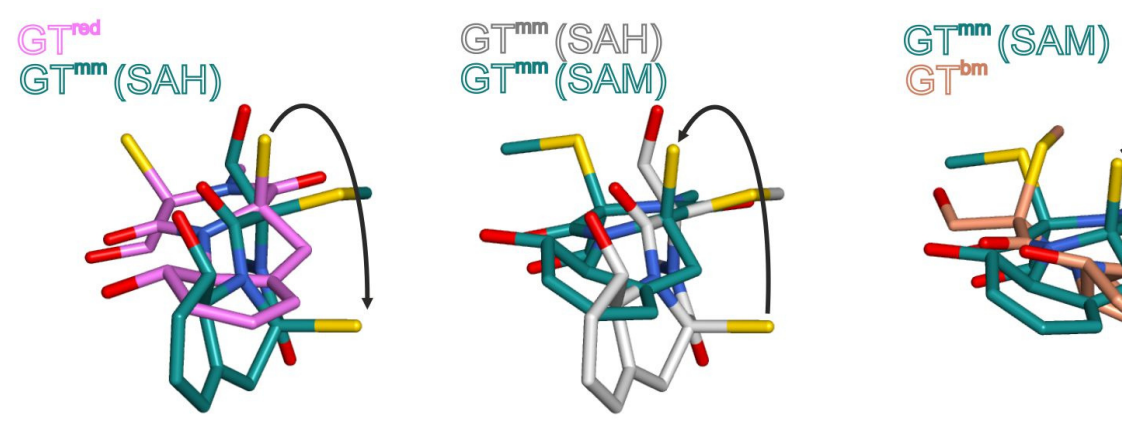

Supporting Figure S9. Alternative $S$-methylation sequence of gliotoxin by TmtA. Initial methylation of the C3-SH-group is followed by alkylation of the C10a-SH site. This Figure corresponds to Figure 3, which illustrates at first methylation of the C10a-SH and secondly modification of the $\mathrm{C} 3-\mathrm{SH}$ group. a) The 3-SH group of reduced gliotoxin (GT ${ }^{\text {red }}$, magenta) is 
located 5.9 $\AA$ apart from the reactive centre of the cofactor SAM (green). b) Monomethylation at the $\mathrm{C} 3-\mathrm{SH}$ site $\left(\mathrm{GT}^{\mathrm{mm}}\right.$, turquoise) would shift the ligand about $90{ }^{\circ} \mathrm{C}$, thereby positioning the C10a-sulfhydryl group far from the cofactor $(7 \AA)$. c) To allow for methylation at the C10a position, the ligand has to flip back in its initial conformation shown in panel a. d) Finally, bis(methylthio)gliotoxin $\left(\mathrm{GT}^{\mathrm{bm}}\right.$, orange) is formed. e) Structural superpositions of modelled reduced (GT ${ }^{\text {red }}$, magenta), mono- $\left(\mathrm{GT}^{\mathrm{mm}}\right.$, turquoise/gray) and bis-thiomethylated gliotoxin (pose 2, GT ${ }^{\mathrm{bm}}$, orange) illustrate conformational changes of the ligands during the C3-C10a $S$-methylation sequence. The structural changes of gliotoxin that are associated with initial methylation of its $\mathrm{C} 3-\mathrm{SH}$ position are unlikely to occur.
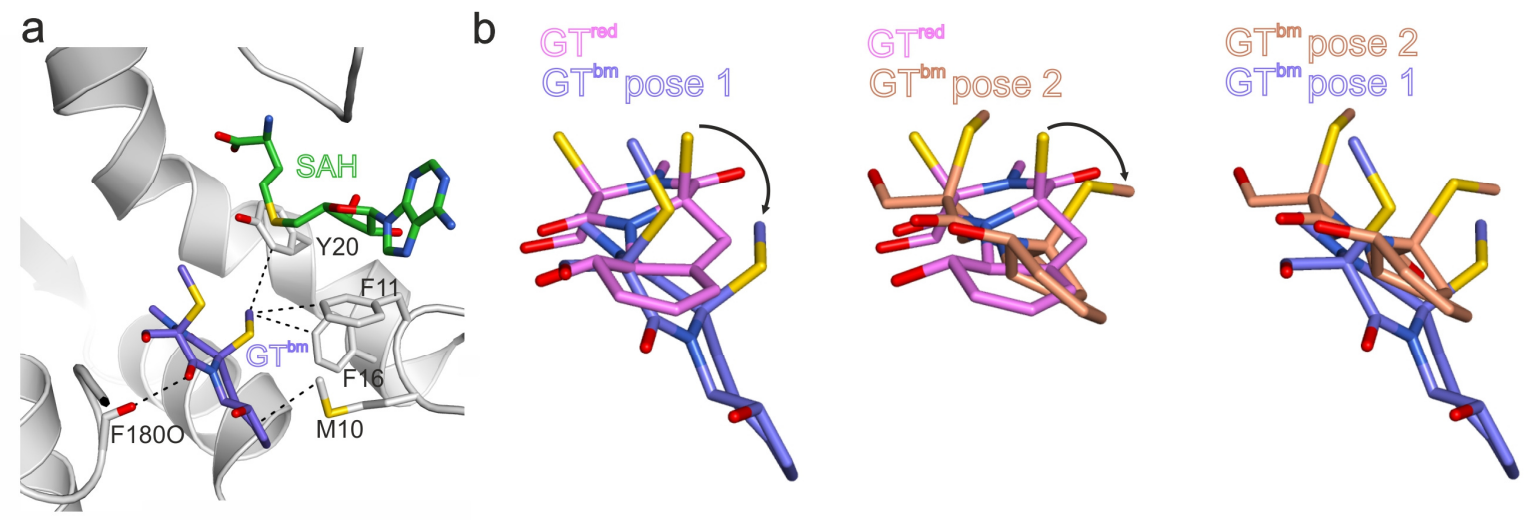

Supporting Figure S10. Alternative docking of bis(methylthio)gliotoxin into the active site of TmtA. Modelling of bis(methylthio)gliotoxin resulted in two alternative conformations, pose 1 and 2 (Table S3). a) Pose 1 of bis(methylthio)gliotoxin (GT ${ }^{\mathrm{bm}}$, purple) bound to TmtA. b) Superposition of reduced gliotoxin $\left(\mathrm{GT}^{\mathrm{red}}\right.$, magenta) and $\mathrm{GT}^{\mathrm{bm}}$ in orientation 1 illustrates profound conformational changes (left) compared to $\mathrm{GT}^{\mathrm{red}}$ superimposed onto pose 2 of $\mathrm{GT}^{\mathrm{bm}}$ (middle). Thus, pose 1 of reduced bis(methylthio)gliotoxin is unlikely. A superposition of poses 1 and 2 of $\mathrm{GT}^{\mathrm{bm}}$ is shown on the right. 


\section{Supporting Tables}

\section{Supporting Table S1. X-ray data collection and refinement statistics}

\begin{tabular}{|c|c|c|}
\hline & TmtA:SAH & $\begin{array}{l}\text { TmtA:SAH } \\
\text { Se-Met peak }\end{array}$ \\
\hline \multicolumn{3}{|l|}{ Crystal parameters } \\
\hline Space group & $\mathrm{P} 2_{1}$ & $\mathrm{P} 2_{1}$ \\
\hline \multirow[t]{4}{*}{ Cell constants } & $\mathrm{a}=49.10 \AA$ & $\mathrm{a}=48.70 \AA$ \\
\hline & $\mathrm{b}=109.21 \AA$ & $\mathrm{b}=109.44 \AA$ \\
\hline & $c=59.94 \AA$ & $c=59.77 \AA$ \\
\hline & $\beta=112.45^{\circ}$ & $\beta=112.3^{\circ}$ \\
\hline $\mathrm{M} / \mathrm{AU}^{\mathrm{a}}$ & 2 & 2 \\
\hline \multicolumn{3}{|l|}{ Data collection } \\
\hline Beam line & X06SA, SLS & X06SA, SLS \\
\hline Wavelength $(\AA)$ & 1.0 & 0.9791 \\
\hline \multirow{2}{*}{ Resolution range $(\AA)^{\mathrm{b}}$} & $45-1.5$ & $45-1.8$ \\
\hline & $(1.6-1.5)$ & $(1.9-1.8)$ \\
\hline No. observations & 279715 & 401642 \\
\hline No. unique reflections ${ }^{c}$ & $89458^{*}$ & $104662^{\#}$ \\
\hline Completeness $(\%)^{\mathrm{b}}$ & $95.9(94.8)$ & $98.7(98.8)$ \\
\hline $\mathrm{R}_{\text {merge }}(\%)^{\mathrm{b}, \mathrm{d}}$ & $5.5(53.0)$ & $5.7(49.9)$ \\
\hline $\mathrm{I} / \sigma(\mathrm{I})^{\mathrm{b}}$ & $14.0(2.8)$ & $15.0(2.6)$ \\
\hline \multicolumn{3}{|l|}{ Refinement (REFMAC5) } \\
\hline Resolution range $(\AA)$ & $15-1.5$ & \\
\hline No. refl. working set & 84984 & \\
\hline No. refl. test set & 4473 & \\
\hline No. non hydrogen & 5071 & \\
\hline No. of ligand atoms & 52 & \\
\hline Solvent $\left(\mathrm{H}_{2} \mathrm{O}, \mathrm{SO}_{4}\right.$, Acetate $)$ & 528 & \\
\hline $\mathrm{R}_{\text {work }} / \mathrm{R}_{\text {free }}(\%)^{\mathrm{e}}$ & $13.2 / 16.4$ & \\
\hline r.m.s.d. bond $(\AA) /\left(^{\circ}\right)^{f}$ & $0.008 / 1.349$ & \\
\hline Average B-factor $\left(\AA^{2}\right)$ & 17.70 & \\
\hline Ramachandran Plot $(\%)^{\mathrm{g}}$ & $99.6 / 0.4 / 0$ & \\
\hline PDB accession code & 5EGP & \\
\hline
\end{tabular}

[a] Asymmetric unit

${ }^{[b]}$ The values in parentheses for resolution range, completeness, $R_{\text {merge }}$ and $I / \sigma$ (I) correspond to the highest resolution shell

${ }^{[c]}$ Data reduction was carried out with XDS and from a single crystal

*Friedel pairs were treated as identical reflections

${ }^{\#}$ Friedel pairs were treated as individual reflections

${ }^{[\mathrm{d}]} \mathrm{R}_{\text {merge }}(\mathrm{I})=\Sigma_{\mathrm{hkl}} \Sigma_{\mathrm{j}}\left|\mathrm{I}(\mathrm{hkl})_{\mathrm{j}}-\langle\mathrm{I}(\mathrm{hkl})\rangle\right| / \Sigma_{\mathrm{hkl}} \Sigma_{\mathrm{j}} \mathrm{I}(\mathrm{hkl})_{\mathrm{j}}$, where $\mathrm{I}(\mathrm{hkl})_{\mathrm{j}}$ is the $\mathrm{j}^{\text {th }}$ measurement of the intensity of reflection $\mathrm{hkl}$ and $\langle\mathrm{I}(\mathrm{hkl})\rangle$ is the average intensity

${ }^{[\mathrm{e}]} \mathrm{R}=\Sigma_{\mathrm{hkl}}|| \mathrm{F}_{\mathrm{obs}}|-| \mathrm{F}_{\text {calc }}|| / \Sigma_{\text {hkl }}\left|\mathrm{F}_{\text {obs }}\right|$, where $\mathrm{R}_{\text {free }}$ is calculated without a sigma cut off for a randomly chosen $5 \%$ of reflections, which were not used for structure refinement, and $\mathrm{R}_{\mathrm{work}}$ is calculated for the remaining reflections

${ }^{[\mathrm{f}]}$ Deviations from ideal bond lengths/angles

${ }^{[\mathrm{g}]}$ Number of residues in favored region / allowed region / outlier region 


\section{Supporting Table S2. Proteins structurally related to TmtA according to the DALI search}

\begin{tabular}{|c|c|c|c|c|}
\hline $\begin{array}{l}\text { PDB entry } \\
\text { code }\end{array}$ & Z-Score & R.m.s.d. $[\AA]$ & Identity [\%] & PDB entry title \\
\hline 2YQZ & 20.3 & 3.3 & 16 & $\begin{array}{c}\text { Crystal Structure of Hypothetical } \\
\text { Methyltransferase TTHA0223 from } \\
\text { Thermus thermophilus HB8 } \\
\text { complexed with } S \text {-adenosylmethionine }\end{array}$ \\
\hline 4OBW & 20.1 & 2.7 & 20 & $\begin{array}{l}\text { Crystal structure of yeast Coq5 in the } \\
\text { SAM bound form }\end{array}$ \\
\hline 3G5T & 20.0 & 3.2 & 13 & $\begin{array}{c}\text { Crystal structure of trans-aconitate } 3 \text { - } \\
\text { methyltransferase from yeast }\end{array}$ \\
\hline $2 \mathrm{P} 35$ & 19.4 & 3.7 & 19 & $\begin{array}{c}\text { Crystal structure of trans-aconitate } \\
\text { methyltransferase from } \\
\text { Agrobacterium tumefaciens }\end{array}$ \\
\hline $3 \mathrm{CCF}$ & 19.3 & 3.2 & 14 & $\begin{array}{c}\text { Crystal structure of putative } \\
\text { methyltransferase (YP_321342.1) } \\
\text { from Anabaena variabilis ATCC } \\
29413 \text { at } 1.90 \text { A resolution }\end{array}$ \\
\hline 1VL5 & 18.6 & 3.8 & 19 & $\begin{array}{c}\text { Crystal structure of a putative } \\
\text { methyltransferase (BH2331) from } \\
\text { Bacillus halodurans } \mathrm{C}-125 \text { at } 1.95 \AA \\
\text { resolution }\end{array}$ \\
\hline $4 \mathrm{KRI}$ & 18.3 & 3.0 & 16 & $\begin{array}{c}\text { Haemonchus contortus } \\
\text { Phospholethanolamine N- } \\
\text { methyltransferase } 2 \text { in complex with } \\
\text { phosphomonomethylethanolamine and } \\
\text { S-adenosylhomocysteine }\end{array}$ \\
\hline $3 \mathrm{DH} 0$ & 18.3 & 2.5 & 21 & $\begin{array}{c}\text { Crystal structure of a SAM dependent } \\
\text { methyltransferase from Aquifex } \\
\text { aeolicus }\end{array}$ \\
\hline $1 \mathrm{XXL}$ & 18.2 & 3.7 & 14 & $\begin{array}{c}\text { Crystal structure of YcgJ protein from } \\
\text { Bacillus subitilis at } 2.1 \AA \\
\text { resolution }\end{array}$ \\
\hline $2 \mathrm{GLU}$ & 18.2 & 3.7 & 14 & $\begin{array}{l}\text { Crystal structure of YcgJ protein from } \\
\text { Bacillus subtilis }\end{array}$ \\
\hline $1 \mathrm{M} 6 \mathrm{E}$ & 18.2 & 4.0 & 11 & $\begin{array}{c}\text { Crystal structure of salicylic acid } \\
\text { carboxyl methyltransferase (SAMT) }\end{array}$ \\
\hline 1IM8 & 18.1 & 2.8 & 15 & $\begin{array}{l}\text { Crystal structure of } \mathrm{YecO} \text { from } \\
\text { Haemophilus influenzae (HI0319), } \\
\text { a methyltransferase with a bound } \\
\text { S-adenosylhomocysteine }\end{array}$ \\
\hline 3DLC & 18.1 & 3.2 & 17 & $\begin{array}{c}\text { Crystal structure of a putative } S \text { - } \\
\text { adenosyl-L-methionine-dependent } \\
\text { methyltransferase (mmp1179) from } \\
\text { Methanococcus maripaludis at } 1.15 \AA \\
\text { resolution }\end{array}$ \\
\hline
\end{tabular}

The DALI server ${ }^{33}$ identified numerous crystal structures of methyltransferases that display 3D similarity to TmtA. The best hits are shown and listed according to their Z-score. 


\section{Supporting Table S3. FlexX scores and free energies of binding of docked ligands and cofactors}

\begin{tabular}{lccc}
\hline Modelled ligand or coenzyme & $\begin{array}{c}\Delta \boldsymbol{G}_{\text {FlexX-Score }} \\
{\left[\mathbf{k c a l ~ m o l}^{-1}\right]^{\mathbf{I}}}\end{array}$ & $\begin{array}{c}\Delta \boldsymbol{G}_{\mathbf{M M - P B S A}} \\
{\left[\mathbf{k c a l ~ m o l}^{-1}\right]}\end{array}$ & $\begin{array}{c}\Delta \boldsymbol{G}_{\mathbf{M M} \text {-GBSA }} \\
{\left[\mathbf{k c a l ~ m o l}^{-1}\right]}\end{array}$ \\
\hline reduced gliotoxin, SAM & -2.56 & -27.54 & -36.32 \\
\hline 3-SH mono(methylthio)gliotoxin, SAH & -1.15 & -24.20 & -31.06 \\
\hline 3-SH mono(methylthio)gliotoxin, SAM & -1.05 & -30.02 & -36.78 \\
\hline 10a-SH mono(methylthio)gliotoxin, SAH & -0.76 & -32.20 & -43.34 \\
\hline 10a-SH mono(methylthio)gliotoxin, SAM & -1.33 & -26.97 & -41.22 \\
\hline bis(methylthio)gliotoxin, SAH, rank 1 & -1.52 & -35.62 & -38.40 \\
\hline bis(methylthio)gliotoxin, SAH, rank 2 & -0.59 & -36.26 & -66.70 \\
\hline SAM & -8.35 & -61.00 & -55.31 \\
\hline SAH & -7.95 & -43.87 & \\
\hline
\end{tabular}

${ }^{1}$ The FlexX scores obtained by the FlexX-Pharm molecular docking studies are very small. Although this is in general in agreement with the high $\mathrm{K}_{\mathrm{M}}$ value obtained experimentally ${ }^{34}$, the differences are by far smaller than the overall accuracy of docking scoring functions $\left(\sim 2 \mathrm{kcal} \mathrm{mol}^{-1}\right)$ and thus not significant. We therefore performed additional MM-PBSA and MM-GBSA molecular dynamics calculations based on the FlexX-Pharm docking poses (columns 2 and 3).

\section{Supporting Table S4. Molecular dynamics equilibration protocol}

\begin{tabular}{|c|c|c|c|c|}
\hline Equilibration Step & $\begin{array}{c}\text { Temperature } \\
{[\mathrm{K}]}\end{array}$ & $\begin{array}{c}\text { Simulation time } \\
\text { [ps] }\end{array}$ & $\begin{array}{l}\text { Force constant } \\
{\left[\mathrm{kcal} \cdot \mathrm{mol}^{-1} \cdot \AA^{-2}\right]}\end{array}$ & Restrained Atoms \\
\hline 1 & 0 & 10 & 2.39 & all solute atoms \\
\hline 2 & 5 & 50 & 2.39 & all solute atoms \\
\hline 3 & 10 & 50 & 2.39 & all solute atoms \\
\hline 4 & 20 & 50 & 2.39 & all solute atoms \\
\hline 5 & 50 & 50 & 2.39 & $\begin{array}{l}\text { protein backbone } \\
\text { heavy atoms }\end{array}$ \\
\hline 6 & 100 & 50 & 2.39 & $\begin{array}{l}\text { protein backbone } \\
\text { heavy atoms }\end{array}$ \\
\hline 7 & 200 & 50 & 2.39 & $\begin{array}{l}\text { protein backbone } \\
\text { heavy atoms }\end{array}$ \\
\hline 8 & 200 & 150 & 0.24 & $\begin{array}{c}\text { protein backbone } \\
\text { heavy atoms }\end{array}$ \\
\hline 9 & 300 & 100 & 0.24 & $\begin{array}{c}\text { protein backbone } \\
\text { heavy atoms }\end{array}$ \\
\hline 10 & 300 & 100 & no restraining force & no restrained atoms \\
\hline
\end{tabular}




\section{Supporting Table S5. Oligonucleotides used for mutagenesis}

\begin{tabular}{ll}
\hline \multicolumn{1}{c}{ Primer } & \multicolumn{1}{c}{$\boldsymbol{5}^{\prime} \boldsymbol{\rightarrow} \mathbf{3}^{\prime}$ Sequence } \\
\hline Y20A_for & GAGCTTTTCGGTGTACTTGGCGCGGTCAACAAAGCTCTTG \\
Y20A_rev & CAAGAGCTTTGTTGACCGCGCCAAGTACACCGAAAAGCTC \\
W162A_for & CGGCTTTCATAATTGGAATCGCGTTGAAGTTCTGCCATGTTG \\
W162A_rev & CAACATGGCAGAACTTCAACGCGATTCCAATTATGAAAGCCG \\
\hline
\end{tabular}

\section{Supporting References}

(1) Rarey, M., Kramer, B., Lengauer, T., and Klebe, G. (1996) A fast flexible docking method using an incremental construction algorithm, J. Mol. Biol. 261, 470-489.

(2) Hindle, S. A., Rarey, M., Buning, C., and Lengauer, T. (2002) Flexible docking under pharmacophore type constraints, J. Comput. Aided Mol. Des. 16, 129-149.

(3) Eleuteri, A. M., Kohanski, R. A., Cardozo, C., and Orlowski, M. (1997) Bovine spleen multicatalytic proteinase complex (proteasome). Replacement of $X, Y$, and $Z$ subunits by LMP7, LMP2, and MECL1 and changes in properties and specificity, J. Biol. Chem. 272, 11824-11831.

(4) Evans, D. A. (2014) History of the Harvard ChemDraw project, Angew. Chem. Int. Ed. Engl. 53, 11140-11145.

(5) Hinchliffe, A. (1996) CS Chem3D Pro (Version 3.2 for Windows), Electron. J. Theor. Chem. 1, 84-86.

(6) DeLano, W. L. (2002) The PyMOL Molecular Graphics System, DeLano Scientific, San Carlos, CA, USA.

(7) O'Boyle, N. M., Banck, M., James, C. A., Morley, C., Vandermeersch, T., and Hutchison, G. R. (2011) Open Babel: An open chemical toolbox, J. Cheminform. 3, 33.

(8) Hartmann, C., Antes, I., and Lengauer, T. (2007) IRECS: a new algorithm for the selection of most probable ensembles of side-chain conformations in protein models, Protein Sci. 16, 1294-1307.

(9) Hartmann, C., Antes, I., and Lengauer, T. (2009) Docking and scoring with alternative side-chain conformations, Proteins 74, 712-726.

(10) Case, D. A., Babin, V., Berryman, J. T., Betz, R. M., Cai, Q., Cerutti, D. S., Cheatham, T. E., III, Darden, T. A., Duke, R. E., Gohlke, H., Goetz, A. W., Gusarov, S., Homeyer, N., Janowski, P., Kaus, J., Kolossváry, I., Kovalenko, A., Lee, T. S., LeGrand, S., Luchko, T., Luo, R., Madej, B., Merz, K. M., Paesani, F., Roe, D. R., Roitberg, A., Sagui, C., Salomon-Ferrer, R., Seabra, G., Simmerling, C. L., Smith, W., Swails, J., Walker, R. C., Wang, J., Wolf, R. M., Wu, X., and Kollman, P. A. (2014) AMBER 14, University of California, San Francisco.

(11) Duan, Y., Wu, C., Chowdhury, S., Lee, M. C., Xiong, G., Zhang, W., Yang, R., Cieplak, P., Luo, R., Lee, T., Caldwell, J., Wang, J., and Kollman, P. (2003) A point-charge force field for molecular mechanics simulations of proteins based on condensed-phase quantum mechanical calculations, $J$. Comput. Chem. 24, 1999-2012.

(12) Wang, J., Wolf, R. M., Caldwell, J. W., Kollman, P. A., and Case, D. A. (2004) Development and testing of a general amber force field, J. Comput. Chem. 25, 1157-1174.

(13) Vanquelef, E., Simon, S., Marquant, G., Garcia, E., Klimerak, G., Delepine, J. C., Cieplak, P., and Dupradeau, F. Y. (2011) R.E.D. Server: a web service for deriving RESP and ESP charges and building force field libraries for new molecules and molecular fragments, Nucleic Acids Res. 39, W511-517.

(14) Wang, J., Wang, W., Kollman, P. A., and Case, D. A. (2006) Automatic atom type and bond type perception in molecular mechanical calculations, J. Mol. Graph. Model. 25, 247-260. 
(15) Schafmeister, C. E. A. F., Ross, W. S., and Romanovski, V. (1995) LEAP, University of California, San Francisco.

(16) Jorgensen, W. L., Chandrasekhar, J., Madura, J. D., Impey, R. W., and Klein, M. L. (1983) Comparison of simple potential functions for simulating liquid water, J. Chem. Phys. 79.

(17) Darden, T., York, D., and Pedersen, L. (1993) Particle mesh Ewald: An N·log(N) method for Ewald sums in large systems, J. Chem. Phys. 98, 10089-10092.

(18) Ryckaert, J.-P., Ciccotti, G., and Berendsen, H. J. C. (1977) Numerical integration of the cartesian equations of motion of a system with constraints: Molecular dynamics of n-alkanes., J. Comput. Phys. 23, 327-341.

(19) Berendsen, H. J. C., Postma, J. P. M., van Gunsteren, W. F., DiNola, A., and Haak, J. R. (1984) Molecular dynamics with coupling to an external bath., J. Chem. Phys. 81, 3684-3690.

(20) Salomon-Ferrer, R., Götz, A. W., Poole, D., Grand, S. L., and Walker, R. C. (2013) Routine Microsecond Molecular Dynamics Simulations with AMBER on GPUs. 2. Explicit Solvent Particle Mesh Ewald, J. Chem. Theory Comput. 9, 3878-3888.

(21) Kollman, P. A., Massova, I., Reyes, C., Kuhn, B., Huo, S., Chong, L., Lee, M., Lee, T., Duan, Y., Wang, W., Donini, O., Cieplak, P., Srinivasan, J., Case, D. A., and Cheatham, T. E., 3rd. (2000) Calculating structures and free energies of complex molecules: combining molecular mechanics and continuum models, Acc. Chem. Res. 33, 889-897.

(22) Srinivasan, J., Cheatham, T. E., III, Cieplak, P., Kollman, P. A., and Case, D. A. (1998) Continuum Solvent Studies of the Stability of DNA, RNA, and Phosphoramidate-DNA Helices, J. Am. Chem. Soc. 120, 9401-9409.

(23) Chong, L. T., Duan, Y., Wang, L., Massova, I., and Kollman, P. A. (1999) Molecular dynamics and free-energy calculations applied to affinity maturation in antibody 48G7, Proc. Natl. Acad. Sci. USA 96, 14330-14335.

(24) Bill R. MillerlII, B. R., McGeeJr., T. D., Swails, J. M., Homeyer, N., Gohlke, H., and Roitberg, A. E. (2012) MMPBSA.py: An Efficient Program for End-State Free Energy Calculations, J. Chem. Theory Comput. 8, 3314-3321.

(25) Tan, C., Yang, L., and Luo, R. (2006) How well does Poisson-Boltzmann implicit solvent agree with explicit solvent? A quantitative analysis, J. Phys. Chem. B 110, 18680-18687.

(26) Onufriev, A., Bashford, D., and Case, D. A. (2004) Exploring protein native states and large-scale conformational changes with a modified generalized born model, Proteins 55, 383-394.

(27) Gohlke, H., Kiel, C., and Case, D. A. (2003) Insights into protein-protein binding by binding free energy calculation and free energy decomposition for the Ras-Raf and Ras-RalGDS complexes, J. Mol. Biol. 330, 891-913.

(28) Weiser, J., Shenkin, P. S., and Still, W. C. (1999) Approximate atomic surfaces from linear combinations of pairwise overlaps (LCPO), J. Comput. Chem. 20, 217-230.

(29) Dai, Y. N., Zhou, K., Cao, D. D., Jiang, Y. L., Meng, F., Chi, C. B., Ren, Y. M., Chen, Y., and Zhou, C. Z. (2014) Crystal structures and catalytic mechanism of the C-methyltransferase Coq5 provide insights into a key step of the yeast coenzyme Q synthesis pathway, Acta Crystallogr. Sect. D - Biol. Crystallogr. 70, 2085-2092.

(30) Lee, S. G., and Jez, J. M. (2013) Evolution of structure and mechanistic divergence in di-domain methyltransferases from nematode phosphocholine biosynthesis, Structure 21, 1778-1787.

(31) Zubieta, C., Ross, J. R., Koscheski, P., Yang, Y., Pichersky, E., and Noel, J. P. (2003) Structural basis for substrate recognition in the salicylic acid carboxyl methyltransferase family, The Plant cell 15, 1704-1716.

(32) Lim, K., Zhang, H., Tempczyk, A., Bonander, N., Toedt, J., Howard, A., Eisenstein, E., and Herzberg, O. (2001) Crystal structure of YecO from Haemophilus influenzae (HI0319) reveals a methyltransferase fold and a bound S-adenosylhomocysteine, Proteins 45, 397-407.

(33) Holm, L., and Rosenstrom, P. (2010) Dali server: conservation mapping in 3D, Nucleic Acids Res. 38, W545-549. 
(34) Scharf, D. H., Habel, A., Heinekamp, T., Brakhage, A. A., and Hertweck, C. (2014) Opposed effects of enzymatic gliotoxin N- and S-methylations, Journal of the American Chemical Society 136, 1167411679. 